\title{
The cost associated with disease-related malnutrition in Ireland
}

\author{
Niamh Rice ${ }^{1, *}$ and Charles Normand ${ }^{2}$ \\ ${ }^{1} N R$ Consulting, 3 Lumiera, Kilmacanogue, Co. Wicklow, Republic of Ireland: ${ }^{2}$ Centre for Health Policy and \\ Management, Trinity College Dublin, College Green, Dublin 2, Republic of Ireland
}

Submitted 12 April 2010: Accepted 12 December 2011: First published online 8 February 2012

\begin{abstract}
Objective: The present study aimed to establish the annual public expenditure arising from the health and social care of patients with diet-related malnutrition (DRM) in the Republic of Ireland.

Design: Costs were calculated by (i) estimating the prevalence of DRM in health-care settings derived from age-standardised comparisons between available Irish data and large-scale UK surveys and (ii) applying relevant costs from official sources to estimates of health-care utilisation by adults with DRM. No attempt has been made to estimate separately the costs of DRM and any associated disease, since each can be a cause or consequence of the other. The methods used are adapted from an evaluation of the cost of malnutrition in the UK by the British Association for Parenteral and Enteral Nutrition (2009).

Settings: Hospitals, nursing homes, out-patient clinics, primary-care clinics and home care.

Subjects: All adult patients receiving hospital in-patient, out-patient or specified community health-care services.

Results: The annual public health and social care cost associated with adult malnourished patients in Ireland is estimated at over $€ 1.4$ billion, representing $10 \%$ of the health-care budget. Most of this cost arises in acute hospital or residential care settings (i.e. 70\%), with nutritional support estimated to account for $<3 \%$ of spend. Conclusions: The cost associated with the care of patients with DRM is substantial and may rise as the proportion of older people within the population increases, a group at increased risk of DRM. Despite growing pressure on health-care budgets, little attention has been focused on the economic burden associated with DRM in Ireland or the potential for savings arising from improved detection and treatment of those at risk.
\end{abstract}

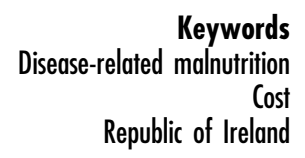

Disease-related malnutrition (DRM) encompasses malnutrition that arises in association with both acute and chronic disease ${ }^{(1)}$. Unlike starvation-related malnutrition, disease may interfere with the ingestion or absorption of nutrients, increase energy needs or cause undernutrition due to significant dietary restrictions imposed during medical treatment ${ }^{(2)}$. Such DRM is a significant public health problem in both primary and secondary healthcare settings, affecting an estimated 20 million people in the European Union at an annual cost of at least $€ 120$ billion $^{(3)}$. Such is the scale and cost of DRM that it is now the subject of pan-European initiatives aimed at tackling the lack of awareness, detection and treatment of this condition by governments, health and social care providers, health-care professionals, patients and carers ${ }^{(4-6)}$. The economic implications of DRM are significant and arise primarily through increased health and social care utilisation, since DRM increases complications of disease, delays recovery, increases length of hospital stay and increases the likelihood that care will be needed after discharge $^{(7-11)}$. Malnourished individuals in the community have also been found to have more visits to general practitioners (GP), out-patient attendances and hospital admissions than well-nourished individuals ${ }^{(9,12-14)}$.

In the UK, the British Association for Parenteral and Enteral Nutrition (BAPEN) estimated the cost of DRM in 2007 to be over $£ 13$ billion, corresponding to more than $10 \%$ of total expenditure on health and social care and more than double the cost of obesity and its co-morbidities ${ }^{(15,16)}$. In the Republic of Ireland there have been few published studies on the prevalence of DRM, and none of its likely costs. This is despite an urgent need to identify areas for potential savings within the Irish health-care system, and to avoid any increase in DRM or its costs that might be predicted to occur as the proportion of the population aged 65 years or more rises from its current level of $11 \%$ to $16 \%$ within the next 15 years ${ }^{(17)}$. Against this background, the present study examines the likely scale of 
DRM in different care settings in Ireland and the associated resource and cost implications for the health service, providing a basis for future strategies aimed at cost reduction.

\section{Methods}

The study aimed to establish the likely costs associated with the health and social care of patients with DRM in Ireland, using the methodology developed by Elia and Stratton to determine the costs of malnutrition in the UK, published as part of a BAPEN report in $2009^{(15)}$.

First, it was necessary to establish the prevalence of DRM in hospitals and the community as a basis for determining the proportion of health and social care resources that might be attributed to patients with DRM. Estimates were established for each care setting using age-standardised comparisons between available Irish data and large-scale UK surveys that employed validated and consistent criteria. These are described in more detail in the subsection entitled 'Estimating the prevalence of diet-related malnutrition'.

Official publications and national data sets provided by the Department of Health and Children, the Health Service Executive, and the Economic and Social Research Institute were used to establish patient activity levels and cost information for hospital and community services in 2007. The methods for calculating total costs per service and the estimated proportion attributable to patients with DRM is outlined in the subsection entitled 'Quantifying the proportion of health and social care used by adults with diet-related malnutrition'.

\section{Estimating the prevalence of diet-related malnutrition}

The prevalence of DRM was estimated for different healthcare settings, i.e. acute hospitals, psychiatric hospitals/ mental health units, hospital out-patient and primary-care (GP) clinics, and in social care, i.e. long-stay care for the elderly (long-stay hospitals, nursing and residential care homes) and for adults receiving care at home. As the prevalence of DRM is highly influenced by the criteria used to define malnutrition, estimates were based on studies which used the Malnutrition Universal Screening Tool (MUST), developed by BAPEN ${ }^{(15,18)}$ (http://www. bapen.org.uk/musttoolkit.html). The MUST screening tool has been validated for use in adults across all healthcare settings and has been shown to predict clinical outcome in different patient groups and to have fair/good to excellent agreement with other validated screening tools ${ }^{(11,18,19)}$. MUST criteria were also used in the largest Irish survey of DRM in hospitals and care homes as part of the BAPEN Nutrition Screening Week Survey (NSW10) conducted in $2010^{(20)}$, allowing direct comparison of prevalence rates with large-scale UK studies. MUST comprises a five-step screening process that attributes an overall risk score to an individual (low risk $=0$, medium risk $=1$, high risk $=\geq 2$ ) on the basis of BMI, recent unplanned weight loss and an acute disease effect. Studies using MUST equate DRM with a medium or high risk score for malnutrition, as in the present study.

\section{Acute hospital in-patients}

Findings from 1602 patients from twenty-seven Irish hospitals revealed a prevalence of DRM according to MUST criteria of $33 \%(25 \% \text { high risk, } 8 \% \text { medium risk })^{(20)}$. The survey (NSW10), conducted in winter as the third of four seasonal surveys coordinated by BAPEN, was the first in which Irish hospitals participated. While the results were similar to those obtained in UK hospitals, they were somewhat higher than the prevalence figures obtained from surveys in $2007(27 \%)$ and $2008(28 \%)^{(21,22)}$. This may reflect a seasonal effect on the type of admissions, which the BAPEN researchers intend to eliminate by amalgamating the results of four surveys to get a more complete picture of DRM over the year. To reduce the possibility that the extrapolation of results from one study to the full year might overestimate annual prevalence, a $10 \%$ reduction has been applied to that obtained (i.e. $33 \% \times 0.9=30 \%$ ), bringing it closer to the mean of the results obtained from amalgamating the three UK surveys ${ }^{(20-22)}$. This is also within the range of prevalence rates of DRM observed in hospitals in other European countries, many of which employed similar criteria to MUST but in some cases different screening tools; $23.8 \%$ in The Netherlands, $25 \%$ in Scotland, $27 \cdot 4 \%$ in Germany, $27 \%$ in Sweden and $41 \%$ in Hungary ${ }^{(23-27)}$.

Patients at risk of DRM (using MUST criteria) have been found to have a $30 \%$ longer length of hospital stay ${ }^{(9)}$. Therefore, the proportion of in-patients with DRM at any time was calculated to be $36 \cdot 3 \%$, using the equation: $p \times[1 \cdot 3-(0 \cdot 3 p)]$, where $p$ equals the proportion of patients at risk of malnutrition on admission (30\%) and 1.3 is the ratio of length of stay in malnourished patients relative to non-malnourished patients. In other words, malnourished patients can be expected to account for at least 36.3\% of adult in-patient bed-days and associated hospital costs.

\section{Psychiatric hospital/mental health unit in-patients}

Psychiatric units in general hospitals and psychiatric hospitals had an average of 3314 in-patients in $2007^{(28)}$, of whom $20 \%$ were in private hospitals ${ }^{(29)}$. As there are no published data on the prevalence of DRM in Irish psychiatric in-patients, a similar prevalence to that found in the BAPEN 2007 and 2008 screening surveys in the UK (overall 19\%) has been assumed ${ }^{(21,22)}$.

\section{Hospital out-patient and primary-care clinics}

Data for the prevalence of DRM in Irish GP clinics are incomplete. A study of Irish adults aged $\geq 65$ years attending a GP clinic found that $15 \%$ had a BMI of $<20 \mathrm{~kg} / \mathrm{m}^{2}$, the-cut off value for BMI risk used in MUST, but the sample size was small $(n$ 48) and no data were 
available on recent weight loss ${ }^{(30)}$. For hospital out-patient and primary-care (GP) clinics, a prevalence of $10 \%$ DRM risk was used by $\mathrm{BAPEN}^{(15)}$ although this is lower than that observed in a recent survey of a range of out-patients in two hospitals in England ( $n$ 321, mean age 54 (SD 16.7) years), which found that $15.9 \%$ were malnourished ${ }^{(31)}$. A recent multi-centre study in The Netherlands ( $n$ 2288, nine centres) found that $7 \%$ of hospital out-patients were malnourished, with the highest prevalence of severe malnutrition of $17 \%$ in oral maxillofacial surgery, $10 \%$ in oncology, $8 \%$ in rehabilitation and $7 \%$ in gastroenterology and pulmonology ${ }^{(32)}$. The figure used by BAPEN for primary care refers to individuals who access the services and not all those who are registered with a GP. However, on the basis that those aged $\geq 65$ years have been found to be at significantly higher risk of DRM than younger adults ${ }^{(22)}$ and as older people account for just $11 \%$ of the Irish population $v$. $16 \%$ of the UK population ${ }^{(33,34)}$, it is estimated that DRM in primary-care settings may be $12 \%$ lower in Ireland. A prevalence of $9 \%$ has therefore been assumed.

\section{Care bomes}

Of 154 residents recently admitted and screened in twelve Irish nursing and residential care homes as part of the BAPEN NSW10, 32\% were malnourished (16\% high risk, $16 \%$ medium risk $)^{(20)}$. This was lower than the $37 \%$ prevalence found in care homes in the UK as part of the same survey (23\% high risk, 15\% medium risk) ${ }^{(20)}$, and may reflect the difference in type of care homes that participated. Furthermore, the survey screened patients within 6 months of admission, despite longer stay having been found to be associated with a higher risk of malnutrition $^{(35)}$. In larger studies that screened all residents in a mixture of nursing and residential homes, risk of malnutrition was found to be 39\% ( $n$ 1176, forty-three care homes) and 32\% ( $n$ 703, nineteen care homes), respectively ${ }^{(36,37)}$. Thus, a prevalence of $35 \%$ across all publicly funded long-stay beds has been assumed.

\section{Care at home}

Since home care packages are made available only to those requiring medium to high caring support to continue to live at home independently ${ }^{(38)}$, and as the majority in receipt of home support are aged $>65$ years, the prevalence of DRM is likely to be significantly higher than in a representative sample of free-living people aged $>65$ years.

A recent study of a group of self-selected Irish older people in receipt of meals-on-wheels at home ( $n$ 63, mean age 78.5 (sD 10.7 ) years) found that although only $2 \%$ were classified as underweight on the basis of a cut-off point of $\mathrm{BMI}<18 \cdot 5 \mathrm{~kg} / \mathrm{m}^{2}, 38.5 \%$ of individuals were assessed as malnourished or at-risk of malnutrition using the Mini Nutritional Assessment ${ }^{(39)}$. This compares with a finding that $31 \%$ of people receiving a delivered meals service in Newcastle, UK were at medium or high risk of DRM using MUST $^{(40)}$, suggesting a prevalence of DRM in those in receipt of home services closer to that found on admission to hospital or care homes than to prevalence estimates for free-living older people ${ }^{(20,41)}$. For the present analysis, a prevalence of $25 \%$ was used for patients receiving home help and/or home care packages, similar to that assumed by the authors of the BAPEN report ${ }^{(15)}$.

\section{Quantifying the proportion of bealth and social care used by adults with diet-related malnutrition}

For in-patient or residential care, costs were estimated by multiplying the total number of publicly funded bed-days, obtained from 2007 records on in-patient activity extracted from the Hospital InPatient Enquiry System (Health Research and Information Division, Economic and Social Research Institute, personal communication, 2009), by the percentage estimated to be malnourished at any time and then by the average bed-day costs obtained from estimates provided by Casemix (Economic and Social Research Institute, personal communication, 2007).

For primary-care (GP) services including general prescription costs, estimates were made of the proportion of total annual spend attributable to malnourished patients and not on activity rates, for which there was insufficient detail. For out-patient visits, the total number of attendances was multiplied by the proportion estimated to be attributable to malnourished patients and by the average cost per attendance. It was assumed that $77 \%$ were publicly funded, in line with overall health-care expenditure split ${ }^{(42)}$.

\section{Costs of in-patient care}

The annual total number of in-patient bed-days for 2007 , excluding those attributable to maternity patients or those aged 0 to 17 years, was $3093122^{(43)}$, of which approximately $76 \%$ were publicly funded ${ }^{(44)}$. Applying the average cost per in-patient day of $€ 844$ (Casemix/Economic and Social Research Institute, personal communication, 2007), this gives a total public cost of adult in-patient care in Ireland of $€ 1.984$ billion. The proportion attributable to patients with DRM was derived from earlier prevalence estimates $(36 \cdot 3 \%)$, without any upward adjustment to allow for higher daily costs of patients with DRM.

\section{Costs of long-term psychiatric care}

The expenditure on long-term residential care in mental health units was $€ 249$ million $^{(45)}$. The proportion of psychiatric-care costs was assumed to be in line with the estimated proportion of patients with DRM (i.e. 19\%).

\section{Cost of hospital out-patients}

The number of out-patient attendances in 2007 (i.e. $\left.3025300^{(46)}\right)$ was multiplied by the average cost per attendance $(€ 160$; Casemix/Economic and Social Research Institute, personal communication, 2007) to obtain a total cost of out-patient care in Ireland of $€ 484$ million, of which $77 \%$ was assumed to be publicly funded (i.e. $€ 373$ million). 
Table 1 Calculation of public expenditure on adult long-stay, residential and nursing home care in Ireland, 2007

\begin{tabular}{lccccc}
\hline Type of bed & $\begin{array}{c}\text { No. of } \\
\text { beds }^{*}\end{array}$ & $\begin{array}{c}\text { Occupancy } \\
\text { rate (\%) }\end{array}$ & $\begin{array}{c}\text { Weekly costt } \\
(€)^{(43)}\end{array}$ & $\begin{array}{c}\text { No. of } \\
\text { weeks }\end{array}$ & $\begin{array}{c}\text { Annual cost } \\
\text { (million } €)\end{array}$ \\
\hline Public long-stay beds & 10545 & 90 & $1227 \ddagger$ & 52 & 606 \\
Private long-stay beds contracted by HSE & 2800 & 90 & $708 \S$ & 52 & 93 \\
Partially public funded long-stay beds by HSE ('sub vented') & 8500 & - & - & - & $171^{*}$ \\
Total annual cost & & & 870 \\
\hline
\end{tabular}

HSE, Health Service Executive.

*Source: Parliamentary Affairs Division, Department of Health and Children, personal communication, 2009.

+Unadjusted for financing, depreciation or return on capital costs.

$\ddagger$ Average of cost of rural homes $(€ 1103)$ and urban homes $(€ 1352)$.

$\S$ A $2 \%$ increase in Consumer Price Index for 2007 applied to 2006 average cost of $€ 694$.

The proportion of attendances by patients with DRM was assumed to be in line with the prevalence estimate for Irish out-patients (i.e. 9\%).

\section{Cost of primary care}

Total Primary Care Reimbursement Services (PCRS) costs for General Medical Services (GMS), including costs for prescriptions and GP services but excluding fees for dental and ophthalmic services, were $€ 2 \cdot 207$ billion $^{(47)}$. Clinical nutrition products cost $€ 53.2$ million (including fees to pharmacists and mark-ups), of which an estimated $€ 13 \cdot 2$ million (PCRS and clinical nutrition manufacturer's data, unpublished results) was spent on specialised gluten-free, low-protein, in-born errors of metabolism and paediatric products, with the remainder (i.e. $€ 40$ million) comprising nutritional support products for adult DRM patients ${ }^{(47)}$. Hence, total GMS expenditure, excluding clinical nutritional products, was $€ 2 \cdot 154$ billion (i.e. $€ 2 \cdot 207$ billion - $€ 0 \cdot 053$ billion). Of these costs, it has been assumed that malnourished patients use $9 \%$ of primary-care costs, in line with earlier prevalence estimates. Although patients with DRM have been shown to have a significantly higher number of GP visits and higher prescription rates than non-malnourished patients ${ }^{(9,12)}$, no attempt has been made to factor this into cost estimates. This is because the majority of GP in Ireland are paid primarily on a capitation fee per person basis and there are insufficient data on the allocation of prescription costs according to age and diagnosis that would allow selective adjustment. For clinical nutrition products, $90 \%$ of all relevant categories of products (tube, sip, specialised) have been assumed to be prescribed for patients with a medium or high risk score for DRM at the time of initial prescription, and $10 \%$ for those at low risk. This is to account for some use of oral supplements for social reasons or due to inappropriate prescribing practice ${ }^{(48)}$.

\section{Public spend on long-stay care for elderly patients}

Total public spend on long-stay nursing and residential home care was calculated from the number and cost of longstay beds for the elderly (see Table 1). The proportion of costs attributable to patients with DRM was calculated from the estimate of DRM prevalence in long-stay facilities.

\section{Other costs not included}

Costs not considered in the present analysis include those for acute day-patient care, day-care services and welfare homes, and those associated with mental health, general dental, audiology, ophthalmic elements, specialist services including rehabilitation and ambulance services.

\section{Sensitivity check}

Increases or decreases of up to $25 \%$ were applied to prevalence estimates according to the degree of uncertainty or likely variance for each care setting.

\section{Results}

Applying estimates of the proportion of health and social care costs attributable to patients with DRM to annual expenditure data for different care settings or services, the total minimum cost associated with DRM patients was calculated to be $€ 1 \cdot 4$ billion ( $€ 1 \cdot 2$ to $€ 1 \cdot 6$ billion, applying sensitivity analyses) based on 2007 activity and costs (Table 2).

\section{Discussion}

The annual public cost of care for patients with DRM in Ireland is estimated at $€ 1.4$ billion, representing $10 \%$ of the $€ 13.7$ billion expenditure on health care in $2007^{(43)}$. This equates to an estimated $€ 1.5$ billion at today's prices, taking into account the higher activity and/or unit costs of hospital and community services, even after allowing for a reduction of approximately $3 \%$ in the number of corresponding in-patient bed-days since $2007^{(49)}$

It should be noted that these calculations make no distinction between the costs of treating DRM and any associated disease. This is because it is difficult to separate the two, since each can be a cause and consequence of the other ${ }^{(15)}$.

Similar to the findings of the BAPEN calculation of the cost of DRM in the UK, this is a minimum value for several reasons ${ }^{(15)}$. First, the costs of several services have not been taken into account (see 'Methods' section) and the total represents public expenditure only, which represented 
Table 2 Calculation of the costs of DRM in Ireland, 2007

\begin{tabular}{|c|c|c|c|c|c|c|c|}
\hline & $\begin{array}{c}\text { Cost } \\
\text { (billion } € \text { ) }\end{array}$ & $\mathrm{pM}$, mean & pM, lower & pM, higher & $\begin{array}{c}\text { Mean } \\
\text { (billion } €)\end{array}$ & $\begin{array}{l}\text { Lower } \\
\text { (billion } €)\end{array}$ & $\begin{array}{c}\text { Upper } \\
\text { (billion } € \text { ) }\end{array}$ \\
\hline \multicolumn{8}{|l|}{ Health care } \\
\hline Hospital in-patients* & 1.984 & 0.363 & 0.309 & $0 \cdot 417$ & $0 \cdot 720$ & 0.613 & $0 \cdot 828$ \\
\hline Specialised psychiatric hospitals & $0 \cdot 249$ & $0 \cdot 190$ & $0 \cdot 152$ & $0 \cdot 228$ & 0.047 & 0.038 & 0.057 \\
\hline Hospital out-patients & 0.373 & 0.090 & 0.072 & $0 \cdot 108$ & 0.034 & 0.027 & 0.040 \\
\hline Primary caret & $2 \cdot 154$ & 0.090 & 0.072 & $0 \cdot 108$ & $0 \cdot 194$ & $0 \cdot 155$ & $0 \cdot 233$ \\
\hline Home nursing services & $0 \cdot 135$ & $0 \cdot 250$ & $0 \cdot 200$ & $0 \cdot 300$ & 0.034 & 0.027 & 0.041 \\
\hline Nutrition support productsł & 0.040 & 0.900 & $0 \cdot 810$ & 0.990 & 0.036 & 0.032 & 0.040 \\
\hline Total of applicable health-care costs & & & & & $1 \cdot 065$ & 0.892 & $1 \cdot 237$ \\
\hline \multicolumn{8}{|l|}{ Welfare/social care } \\
\hline Adult nursing/residential care & $0 \cdot 870$ & $0 \cdot 35$ & 0.28 & 0.42 & 0.304 & 0.243 & 0.365 \\
\hline Adult home help and care packages & 0.207 & $0 \cdot 25$ & $0 \cdot 20$ & $0 \cdot 30$ & 0.052 & 0.041 & 0.062 \\
\hline Total of applicable social care costs & & & & & 0.356 & 0.284 & $0 \cdot 427$ \\
\hline Total & & & & & $1 \cdot 421$ & $1 \cdot 176$ & $1 \cdot 665$ \\
\hline
\end{tabular}

DRM, diet-related malnutrition; pM, proportion malnourished.

${ }^{*}$ Acute hospital in-patient cost excluding maternity based on bed-days $\times$ average bed-day costs $\times$ proportion publicly funded.

tPrimary-care services include general medical and prescriptions but exclude general dental/ophthalmic/nutrition support services.

fHigher and lower values $( \pm 10 \%)$ were established to cover likely range of prescribing for those with low risk of DRM due to limited information.

Includes long-stay hospital bed costs (public spend only) based on $90 \%$ occupancy, and costs of contracted and sub vented private nursing home beds.

Higher and lower values ( \pm 20 and $\pm 25 \%$ ) were established to cover the likely range of DRM prevalence and to take account of the limited information on prevalence of DRM in certain care settings.

just $77 \%$ of total health-care costs in $2007^{(42)}$. Second, we have only included costs associated with DRM in adult patients, whereas DRM affects patients of all ages. We have also excluded maternity and obstetric patients from the data set for hospital in-patients, a group that accounted for nearly $10 \%$ of bed-days, because we had no data on DRM in this group. Third, while the daily cost of care for a malnourished patient is likely to be greater than the cost of caring for a non-malnourished individual, the model used has assumed the costs to be the same ${ }^{(15)}$. Neither have we factored in the increased utilisation of health-care resources by malnourished compared with non-malnourished patients other than in the hospital setting. Finally, 2007 costs and activity levels have been used to obtain as complete a picture as possible, which takes no account of subsequent increases in activity levels and costs associated with hospital and community care between 2007 and $2010^{(49)}$.

There are several limitations of the present study. Whereas the model relies heavily on determining a reliable estimate of prevalence for each care setting using consistent methodology, large-scale Irish studies using MUST were available only for hospital in-patients, necessitating age-adjusted comparison with or extrapolation from UK studies for other settings. The reliance on prevalence data to determine the proportion of costs attributable to the care or treatment of DRM is also a limitation, since it has been well established that malnourished patients use more health-care resources than non-malnourished patients. A recurrent problem with health economic assessments of DRM is the difficulty in extricating the clinical and economic impact of DRM from that of any associated underlying disease or co-morbidity. Studies which have attempted to evaluate the additional costs of treating malnourished $v$. well-nourished patients have based their calculations on differences in health- care utilisation between age- and/or diagnosis-matched groups $^{(9,13)}$. No database of patient records exists in Ireland which would provide the necessary information for such an evaluation, which would ideally include information on patient demographics, diagnosis, co-morbidities, health-care utilisation (i.e. GP visits, out-patient visits, hospital admissions, length of stay, procedures and prescriptions) and nutritional parameters. However, a 2005 BAPEN report in the UK estimated that this represented the majority (i.e. $>70 \%$ ) of the total cost of treating patients with $\mathrm{DRM}^{(9)}$, based on the same criteria used in the present study. If the same relationship can be anticipated to hold for the corresponding health-care elements of this estimate that BAPEN considered in their 2005 report (i.e. in-patient care, long-term care, out-patient visits and nutritional support, excluding GP visits and prescription costs for which precise information was unavailable), the additional cost of care for malnourished $v$. well-nourished in Ireland is likely to exceed $€ 750$ million.

There is accumulating evidence of significant cost savings associated with timely nutritional intervention in both hospital and community patients. A retrospective cost analysis by Stratton et $a l^{(7)}$ of nine randomized controlled trails (with or without oral nutrition supplementation) demonstrated mean cost savings of between $£ 352$ and $£ 8179$ per patient in surgical, orthopaedic, elderly and cerebrovascular accident patients, using significantly lower bed-day costs than apply currently in Ireland. Studies in community patients have also shown net savings associated with oral nutrition supplementation use $v$. standard care in France ${ }^{(50)}$, The Netherlands ${ }^{(51)}$ and Germany ${ }^{(52)}$.

While it is not possible to determine precisely how much of the additional cost of DRM can be saved through an effective screening and intervention strategy in Ireland, this estimate can be used to indicate how much it would be 
worth for a health-care system to spend per patient on successful interventions.

It is estimated that there are approximately 140000 adults with DRM in Ireland at any time. This estimate is based on age-adjusted extrapolations from UK studies in community settings (i.e. $13 \cdot 9 \%$ of those aged $>65$ years and $2 \cdot 5 \%$ of those aged $19-64$ years ${ }^{(41,53)}$ ), added to the number of Irish hospital in-patients with DRM estimated from the NSW10 survey (see 'Methods' section). As the proportion of underweight subjects (i.e. BMI $<18.5 \mathrm{~kg} / \mathrm{m}^{2}$ ) was similar between age-matched groups surveyed as part of the Irish national Survey of Lifestyle, Attitudes and Nutrition (J Lutomski, personal communication, 2009) and the National Dietary and Nutrition Survey in the $\mathrm{UK}^{(53)}$, it was considered reasonable to assume the prevalence of DRM to be similar within the UK and Irish populations.

Based on these assumptions, the additional cost of DRM per adult patient (i.e. $€ 750$ million $\div 140000$ ) is estimated at $€ 5357$ from which we can deduce that any spend below this figure on interventions that successfully prevent or treat DRM might be anticipated to deliver savings. Interventions that cost more may add value to the health-care system by improving the quality of health care but would require justification.

\section{Conclusions}

Given the very high costs associated with DRM, even a small percentage reduction in the prevalence or severity of DRM has the potential to deliver substantial savings within the overall health-care budget, since much DRM is preventable or treatable with nutritional interventions. While hospitals provide an opportunity to identify those at risk and initiate treatment which can be continued in the community, a screening and early intervention programme in the community may well be the most effective means of achieving cost savings. This is because malnourished patients are at significantly increased risks of complications, hospitalisation and longer length of stay once in hospital ${ }^{(7,10,11)}$, and earlier identification and appropriate intervention in the community might be expected to reduce costs by prevention of co-morbidities arising directly as a result of DRM. However, achieving overall savings requires a cross-sectoral approach to budget targeting, since it is likely that savings derived from the prevention and earlier treatment of DRM will be made in hospital and long-term care settings, but may require increases in expenditure on nutritional support from community budgets.

\section{Acknowledgements}

This research received no specific grant from any funding agency in the public, commercial or not-for-profit sectors. There are no conflicts of interest. N.R. collected and analysed the data, adapted the methodology for use from
BAPEN and wrote the paper; C.N. provided advice on methodology and guidance on health economic aspects. The authors are indebted to Professor Marinos Elia for his helpful inputs and guidance, and would like in particular to acknowledge the use of cost models and templates developed by Professor Elia and Dr Rebecca Stratton as the basis for the present study. The authors give special thanks to Fionna Page for her work in reviewing the manuscript and providing editorial assistance.

\section{References}

1. Jensen GL, Mirtallo J, Compher C et al. (2010) Adult starvation and disease-related malnutrition: a proposal for etiology-based diagnosis in the clinical practice setting from the International Consensus Guideline Committee. J Parenter Enteral Nutr 34, 156-159.

2. Institute of Medicine, Food and Nutrition Board (2000) The Role of Nutrition in Maintaining Health in the Nation's Elderly: Evaluating Coverage of Nutrition Services for the Medicare Population. Washington, DC: National Academies Press.

3. Ljungqvist O, van Gossum A, Sanz M et al. (2010) The European fight against malnutrition. Clin Nutr 29, 149-150.

4. Council of Europe, Committee of Ministers (2003) Resolution ResAP (2003)3 on food and nutritional care in hospitals. Strasbourg: Council of Europe; available at https://wcd. coe.int/ViewDoc.jsp?id $=85747$

5. Committee of Experts on Nutrition, Food and Consumer Health (2009) Nutrition in Care Homes and Home Care. Report and Recommendations: From Recommendations to Action. Strasbourg: Council of Europe Publishing.

6. Prague Declaration (2009) Stop Disease Related Malnutrition and diseases due to malnutrition. http://www.europeannutrition.org/record.jsp?type $=$ publication $\& I D=29$ (accessed April 2010).

7. Stratton RJ, Green CJ \& Elia M (2003) Disease-related Malnutrition: An Evidence Based Approach to Treatment. Wallingford: CABI Publishing.

8. Elia M \& Russell C (2009) Combating Malnutrition: Recommendations for Action. Report from the Advisory Group on Malnutrition, Led by BAPEN. Redditch: BAPEN.

9. Elia M, Stratton RJ, Russell C et al. (2005) The Cost of Disease-Related Malnutrition in the UK and Economic Considerations for the Use of Oral Nutritional Supplements (ONS) in Adults. Redditch: BAPEN.

10. Sorensen J, Kondrup J, Prokopowicz J et al. (2008) EuroOOPS: an international, multicentre study to implement nutritional risk screening and evaluate clinical outcome. Clin Nutr 27, 340-349.

11. Stratton RJ, King CL, Stroud MA et al. (2006) 'Malnutrition Universal Screening Tool' predicts mortality and length of hospital stay in acutely ill elderly. Br J Nutr 95, 325-330.

12. Martyn CN, Winter PD, Coles SJ et al. (1998) Effect of nutritional status on use of health care resources by patients with chronic disease living in the community. Clin Nutr 17, 119-123.

13. Guest JF, Panca M, Baeyens JP et al. (2011) Health economic impact of managing patients following a communitybased diagnosis of malnutrition in the UK. Clin Nutr 30, 422-429.

14. Feldblum I, German L, Bilenko N et al. (2009) Nutritional risk and health care use before and after an acute hospitalization among the elderly. Nutrition 25, 415-420.

15. Elia M \& Stratton RJ (2009) Calculating the cost of diseaserelated malnutrition in the UK in 2007 (public expenditure only). In Combating Malnutrition: Recommendations for 
Action. Report from the Advisory Group on Malnutrition, Led by BAPEN. Redditch: BAPEN.

16. House of Commons Health Committee (2004) Obesity: Third Report of Session 2003-04. vol. 1. London: The Stationery Office.

17. Central Statistics Office (2008) Population and Labour Force Projections 2011-2041. http://www.cso.ie/releases publications/po_lab_project.htm (accessed April 2010).

18. Elia M (2003) Screening for Malnutrition: A Multidisciplinary Responsibility. Development and Use of the Malnutrition Universal Screening Tool ('MUST') for Adults. Redditch: BAPEN.

19. Stratton RJ, Hackston A, Longmore D et al. (2004) Malnutrition in hospital outpatients and inpatients: prevalence, concurrent validity and ease of use of the "malnutrition universal screening tool' ('MUST') for adults. Br J Nutr 92, 799-808.

20. Russell C \& Elia M (2011) Nutrition Screening Week in the UK and Republic of Ireland in 2010. Hospitals, Care Homes and Mental Health Units. Redditch: BAPEN.

21. Russell C \& Elia M (2008) Nutrition Screening Survey and Audit of Adults on Admission to Hospitals, Care Homes and Mental Health Units. Redditch: BAPEN.

22. Russell C \& Elia M (2009) Nutrition Screening Survey in the UK in 2008: Hospitals, Care Homes and Mental Health Units. Redditch: BAPEN.

23. Meijers JM, Schols JM, van Bokhorst-de van der Schueren MA et al. (2009) Malnutrition prevalence in The Netherlands: results of the annual Dutch national prevalence measurement of care problems. Br J Nutr 101, 417-423.

24. Ruxton $\mathrm{CH}$, Gordon J, Kirkwood L et al. (2008) Risk of malnutrition in a sample of acute and long-stay NHS Fife in-patients: an audit. J Hum Nutr Diet 21, 81-90.

25. Pirlich M, Schutz T, Norman K et al. (2006) The German hospital malnutrition study. Clin Nutr 25, 563-572.

26. Westergren A, Lindholm C, Axelsson C et al. (2008) Prevalence of eating difficulties and malnutrition among persons within hospital care and special accommodations. $J$ Nutr Health Aging 12, 39-43.

27. Lelovics Z, Bozo-Kegyes R, Bonyar-Muller K et al. (2008) Results of nutritional risk screening of patients admitted to hospital. Acta Physiol Hung 95, 107-118.

28. Mental Health Commission (2008) Annual Report 2008 Including the Report of the Inspector of Mental Health Services. Dublin: Mental Health Commission.

29. Daly A, Walsh D \& Moran R (2008) Health Research Board Statistics Series 5: Activities of Irish Psychiatric Units and Hospitals 2007. Dublin: Health Research Board.

30. Corish CA, Flood P \& Kennedy NP (2000) Nutritional status of the Irish elderly. Ir J Med Sci 169, Suppl. 2, 33A.

31. Rust S, Cawood AL, Walters E et al. (2010) Prevalence of malnutrition in hospital outpatients. Proc Nutr Soc 69, E150.

32. Leistra E, Neelemaat F, Evers AM et al. (2009) Prevalence of undernutrition in Dutch hospital outpatients. Eur J Intern Med 20, 509-513.

33. Central Statistics Office (2007) Census 2006: Principal Demographic Results. Table 5. Persons by Age, Sex and Marital Status. Dublin: Stationery Office; available at http:// www.cso.ie/census/documents/Amended\%20Final\%20 Principal\%20Demographic\%20Results\%202006.pdf

34. Office for National Statistics (2002) Census 2001. http:// www.statistics.gov.uk/census2001/pyramids/pages/UK.asp (accessed April 2010).

35. Suominen M, Muurinen S, Routasalo P et al. (2005) Malnutrition and associated factors among aged residents in all nursing homes in Helsinki. Eur J Clin Nutr 59, 578-583.
36. Parsons EL, Stratton RJ \& Elia M (2010) An audit of the use of oral nutritional supplements in care homes in Hampshire. Proc Nutr Soc 69, E197.

37. Cawood AL, Smith A, Dalrymple-Smith J et al. (2008) Prevalence of malnutrition and use of nutritional support in Peterborough Primary Care Trust. J Hum Nutr Diet 21, 384

38. Health Service Executive (2011) Home care packages. http:// www.hse.ie/eng/services/Find_a_Service/Older_People_ Services/Benefits_and_Entitlements/Home_Care_Packages. html (accessed July 2011).

39. O'Dwyer C, Corish CA \& Timonen V (2009) Nutritional status of Irish older people in receipt of meals-on-wheels and the nutritional content of meals provided. J Hum Nutr Diet 22, 521-527.

40. Skinner RA, Dugdale C, Crowe C et al. (2010) Tackling malnutrition in the community, by review of the hot delivered meals service. Proc Nutr Soc 69, OC49 (abstract).

41. Elia M \& Stratton RJ (2005) Geographical inequalities in nutrient status and risk of malnutrition among English people aged 65 y and older. Nutrition 21, 1100-1106.

42. Organisation for Economic Co-operation and Development (2011) OECD Health Data 2011. How Does Ireland Compare. http://www.oecd.org/dataoecd/45/53/43216301.pdf (accessed July 2011).

43. McEnery B (2007) Nursing Home Cost of Care: A fair price - Ireland. Limerick: Howarth, Bastow, Charlton.

44. Health Research and Information Division, Economic and Social Research Institute (2009) Activity in Acute Public Hospitals in Ireland, Annual Report 2007. http://www. esri.ie/health_information/latest_hipe_nprs_reports/2007/ (accessed April 2010).

45. Health Service Executive (2008) The Efficiency and Effectiveness of Long-Stay Residential Care for Adults within the Mental Health Services: Evaluation report prepared under the Value for Money and Policy Review Initiative. Dublin: HSE.

46. Health Service Executive (2007) Annual Report and Financial Statement. Dublin: HSE.

47. Health Service Executive (2007) Finance Shared Services, Primary Care Reimbursement Service. Statistical Analysis of Claims and Payments 2007. Dublin: HSE.

48. Kennelly S, Kennedy NP, Rughoobur GF et al. (2009) The use of oral nutritional supplements in an Irish community setting. J Hum Nutr Diet 22, 511-520.

49. Health Service Executive (2011) Annual Report and Financial Statements 2010. http://www.hse.ie/eng/services/Publications/ corporate/annualrpt $\% 202010$.html (accessed July 2011).

50. Arnaud-Battandier F, Malvy D, Jeandel C et al. (2004) Use of oral supplements in malnourished elderly patients living in the community: a pharmaco-economic study. Clin Nutr 23, 1096-1103.

51. Nuijten M \& Freyer K (2010) The budget impact of oral nutritional supplements in the community setting in The Netherlands. Value in Health 13, A101 (PHP104; abstract).

52. Nuijten M (2010) The health economic impact of oral nutritional supplements in Germany. Clin Nutr 5, Suppl. 2, 159 (PP347; abstract).

53. Ruston D, Hoare J, Henderson L et al. (2004) The National Diet and Nutrition Survey: Adults Aged 19 to 64 Years. vol. 4: Nutritional Status (Anthropometry and Blood Analyses), Blood Pressure and Physical Activity. London: The Stationery Office.

54. Department of Health and Children (2007) Long Stay Activity Statistics. http://www.dohc.ie/publications/pdf/long_ stay_2007.pdf (accessed August 2009). 